\title{
A novel gene expression system for detecting viable bladder cancer cells
}

\author{
HIDEO UEKI $^{1,2}$, MASAMI WATANABE ${ }^{1-3}$, HARUKI KAKU $^{1-3}$, PENG HUANG $^{1}$, SHUN-AI LI $^{1}$, \\ KAZUHIKO OCHIAI ${ }^{3}$, TAKESHI HIRATA ${ }^{2}$, HIROFUMI NOGUCHI ${ }^{4}$, HIROSHI YAMADA $^{5}$, \\ KOHJI TAKEI $^{5}$, YASUTOMO NASU ${ }^{1-3}$, YUJI KASHIWAKURA ${ }^{3}$ and HIROMI KUMON ${ }^{1-3}$ \\ ${ }^{1}$ Center for Gene and Cell Therapy, ${ }^{2}$ Department of Urology, ${ }^{3}$ Innovation Center Okayama for Nanobio-Targeted \\ Therapy, ${ }^{4}$ Department of Gastroenterological Surgery, ${ }^{5}$ Department of Neuroscience, Graduate School of \\ Medicine, Dentistry and Pharmaceutical Sciences, Okayama University, Okayama 700-8558, Japan
}

Received December 14, 2011; Accepted February 3, 2012

DOI: $10.3892 /$ ijo.2012.1417

\begin{abstract}
A novel transcriptional system was developed that can robustly enhance cancer-specific gene expression. In the system, hTERT promoter-driven gene expression was enhanced by an advanced two-step transcriptional amplification (TSTA). This construct was used to develop a novel system for detection of bladder cancer cells. The current study evaluated the advanced TSTA system by examining the cancer-specific gene transcription in various bladder cancer cell lines. The system significantly enhanced cancer-specific luciferase gene expression in the bladder cancer cell lines in comparison to the previous expression system of one-step or conventional TSTA. The fold gain of the enhancement was significantly correlated to the telomerase activity of the cell lines. A green fluorescent protein (GFP) gene encoding plasmid vector was constructed where hTERT promoter-driving transcription is enhanced by the advanced TSTA to utilize the system for the imaging and detection of viable bladder cancer cells. The advanced TSTAhTERT-GFP plasmid successfully induced cancer-specific gene expression, showing robust GFP expression in human bladder cancer cell lines, but no visible GFP expression in normal bladder urothelial cells. The control GFP plasmid with a CMV promoter yielded GFP expression in both normal bladder cells and cancer cells. The advanced TSTA-hTERT-GFP plasmid allowed selective visualization of viable human bladder cancer cells in mixed cell culture containing 10- and 100-fold more normal bladder urothelial cells. These findings indicate that the advanced TSTA-hTERT expressional system is a valuable tool for detecting viable bladder cancer cells. The current system
\end{abstract}

Correspondence to: Dr Masami Watanabe, Department of Urology, Okayama University, 2-5-1 Shikata-cho, Okayama 700-8558, Japan E-mail: mwcorrespondence@gmail.com

Key words: bladder cancer, human telomerase reverse transcriptase, two-step transcriptional amplification, green fluorescent protein, plasmid can be applied for in vitro detection of bladder cancer cells in urine and other types of cancer cells disseminated in vivo.

\section{Introduction}

Bladder cancer is one of the most common malignancies worldwide. It is the fourth most common malignancy among men in Western countries, following prostate, lung and colon cancers and represents the second most common cause of death among genitourinary tumors (1). Bladder cancer is a heterogeneous group of tumors that display a broad clinical spectrum ranging from superficial and well differentiated lesions to invasive and poorly differentiated cancers. The early detection and diagnosis of bladder cancer is of particular importance in clinical medicine. Cystoscopy is considered the gold standard as a diagnostic tool for bladder cancer while small tumors and carcinoma in situ is sometimes hard to detect by cystoscopy. Urine cytology is often used for the additional examination with high specificity, but the sensitivity is insufficient, especially for the detection of well differentiated tumors. Therefore, some patients show a false-negative diagnosis in spite of a significant disease and it is thus necessary to develop novel diagnostic methods for bladder cancer.

Transcriptional targeting by tissue-specific promoters has been frequently used to limit the expression to the tissue of interest. The human telomerase reverse transcriptase (hTERT) promoter is well characterized and available for cancer tissuespecific gene expression in a broad range of malignant tissue types (2-4). Telomerase has been also proposed as a reliable marker of bladder malignancies because it is detected in about $90 \%$ of transitional cell carcinomas $(5,6)$. The analysis of telomerase activity by hTERT promoter-dependent gene expression seems to be promising examination for the detection of bladder cancer cells in urine (3,6-9). However, the use of the hTERT promoter-driving system for the analysis of telomerase activity is often limited because the promoters cannot lead to sufficient levels of reporter gene expression (9-11). Therefore, the enhancement of the cancer-specific expression is required. We recently developed an advanced two-step transcriptional amplification (TSTA) system to robustly enhance cancerspecific gene expression driven by an hTERT promoter (9-11). 
In the advanced TSTA system, the polyglutamines and rat glucocorticoid receptor (GR) sequences were inserted within the GAL4-VP16 fusion sequence of the conventional TSTA system and this provided a significant transcriptional improvement in comparison to the conventional system $(9,11,12)$. Based on the availability of the hTERT-dependent advanced TSTA system, we newly developed a novel imaging system to detect human bladder cancer cells. This study evaluated this novel system in terms of the enhancement of cancer-specific gene expression in human bladder cancer cells. The study also demonstrated the utility of this system for the in vitro imaging and detection of bladder cancer cells.

\section{Materials and methods}

Cell culture. Normal human bladder urothelial cells (HUC) were purchased from Lonza (Basel, Switzerland) and cultivated using the medium recommended by the supplier. Human bladder cancer cell lines (KK47, HT1376, UMUC3, TCCsup, J82, 5637) were provided by the American Type Culture Collection (Rockville, MD). Dulbecco's modified Eagle's medium (DMEM; Invitrogen, Carlsbad, CA) and RPMI-1640 medium (Nissui, Tokyo, Japan) were used for the culture of human bladder cancer cell lines. The cell lines were grown in media supplemented with $10 \%$ fetal bovine serum (FBS; Biowest, Nuaille, France), as previously described (13).

Mechanism of the enhanced gene expression by advanced TSTA system. The VP16 immediate early transactivator contains a highly potent activation domain that, when fused to the GAL4 binding domain, elicits a robust response on a GAL4-responsive promoter (an adenovirus minimal promoter) bearing multiple tandem copies of the $17 \mathrm{bp}$ of the GAL4 binding site (11). This system is here referred to as the TSTA (two-step transcriptional amplification). For the purpose of improving the conventional TSTA system, we constructed an advanced TSTA system. In the advanced system, we inserted the polyglutamines and rat glucocorticoid receptor (GR) sequences within the GAL4-VP16 fusion sequence, which were previously reported to enhance gene transcription (12). By combining the conventional TSTA system and the sequence of polyglutamines and rat GR, we established a novel hTERT promoter-dependent advanced TSTA system with strong transactivating properties due to the GAL4-VP16 fusion protein and GAL4 binding sites.

Construction of plasmid vectors. The construction of the expressional plasmid vectors (one-step, conventional and advanced TSTA) was described previously (9). Briefly, the hTERT promoter region from -377 to +77 (2) was synthesized and inserted into the pGL3-basic vector (Promega, Madison, WI) at the restriction site of $M l u I-B g l I I$. The GAL4-VP16 fusion sequence (10) and 5x repeated GAL4 binding sites with an adenoviral minimal promoter (11) were inserted into the one-step plasmid at the restriction sites of BglII-HindIII and HindIII-NcoI, respectively, to construct the conventional two-step (TSTA) plasmid. The GAL4-VP16 fusion protein contains the GAL4 binding domain amino acids 1-147 and the N-terminal portion of the VP16 activation domain amino acids 413-456 (10,14). The sequence of polyglutamines and rat GR of the following amino acids was inserted between the GAL4 and VP16 sequences of the conventional two-step plasmid: \{MGLY (37Q)MGETETKVMGNDLGYPQQGQLGLSSGETDRLLE ESIANLNRS\}, to generate the advanced two-step (TSTA) plasmid.

The cDNA encoding green fluorescent protein (GFP) was inserted into the advanced TSTA system to create the advanced TSTA-hTERT-GFP plasmid. The cDNA was obtained from a commercial CMV promoter-GFP plasmid vector by digestion with restriction enzymes and the plasmid vector was also used as a control GFP plasmid in the in vitro transfection of normal human bladder urothelial cells.

Cell transfections and luciferase assays. The cells were plated in 24-well plates in culture medium containing charcoal-stripped FBS. The cells were cultured for $24 \mathrm{~h}$, and transient transfections of the luciferase- or GFP-encoding plasmid with the one-step, conventional TSTA or advanced TSTA system were performed by using the Lipofectamine transfection reagent (Invitrogen). GFP expression was analyzed by fluorescent microscopy $24 \mathrm{~h}$ after the transfection. In the luciferase assay, the effector plasmid was co-transfected with the reporter plasmid derived from the dual-reporter luciferase assay kit (Promega). Each transfection mix contained $0.5 \mu \mathrm{g}$ of the effector and reporter plasmids or the reporter plasmid alone. The cells were incubated for $48 \mathrm{~h}$, harvested and assayed for luciferase activity by using the luciferase assay kit and a luminescence microplate reader, according to the manufacturer's instructions.

Telomerase activity assays. Telo TAGGG Telomerase PCR ELISAPLUS kit (Roche, Basel, Switzerland) was employed according to the manufacturer's instructions. The assay uses the internal activity of telomerase, amplifying the product by PCR and detecting it with an enzyme linked immunosorbent assay. Typically, $2 \times 10^{5}$ cells per sample were harvested, and the pelleted cells were resuspended in $200 \mu \mathrm{l}$ of the lysis reagent. After centrifugation, the supernatants of lysates were collected and $3 \mu \mathrm{l}$ of cell extract (corresponding to $3 \times 10^{3}$ cell equivalents) was used for the following procedures.

In vitro detection of GFP-expressing bladder cancer cells. The experimental system shown in Fig. 4A was developed to test the utility of the advanced TSTA mediated cancer-specific gene expression for detecting human bladder cancer cells. The target bladder cancer KK47 cells were pretreated with the Cell Tracker Orange (Invitrogen) as a tracer according to the manufacturer's instructions. The KK47 cancer cells were then trypsinized and mixed with normal human bladder urothelial cells at different target frequency ratio (1:10 and 1:100, respectively). The mixed cells were cultured for $24 \mathrm{~h}$ and transfected with the advanced TSTA-hTERT-GFP plasmid. The cells were examined for GFP expression and Cell Tracker Orange staining after $24 \mathrm{~h}$ using fluorescent microscopy.

Statistical analysis. The data are presented as the means \pm standard deviation. The unpaired Student's t-test was used to analyze the statistical significance of differences between any 2 groups. A regression analysis was performed to examine the correlation of 2 parameters. Differences or correlations were considered to be significant at $\mathrm{p}<0.05$. 


\section{Results}

The advanced TSTA system mediates hTERT-dependent selective luciferase expression in human bladder cancer cells. The advanced TSTA system was constructed by inserting the polyglutamines and rat glucocorticoid receptor sequences between the GAL4 and VP16 sequences of the region of the first step transcription (Fig. 1A). The telomerase activity was measured using commercially-available kits. Relatively strong telomerase activity was observed in the bladder cancer cell lines, but the level was undetectable in the normal human bladder urothelial cells (HUC) (Fig. 1B). A luciferase assay of the advanced TSTA plasmid in the various cell lines, including normal HUC cells was performed to determine whether the advanced TSTA system-mediated amplification is restricted to the cancer cells with higher telomerase activity. The luciferase expression level was minimal in the normal HUC cells, but more luciferase expression was observed as the telomerase activity increased in the cancer cells. The advanced TSTA system demonstrated a significant correlation between the telomerase activity and the values of luciferase expression (Fig. 1C). Telomerase activity is strongly correlated to the hTERT expression level and hTERT promoter activity (2), thus the advanced TSTA system-mediated gene expression is regulated by the hTERT promoter and is therefore highly bladder cancer-specific.

The advanced TSTA system significantly enhances in vitro gene expression in comparison to both the one-step and conventional TSTA systems. The advanced TSTA system was evaluated as a method to enhance the transcriptional activity of the hTERT promoter in various bladder cancer cell lines. In the luciferase assay, there was a significant enhancement of gene expression using the advanced TSTA approach in comparison to that induced by the one-step and conventional TSTA systems (Fig. 2A). There was a 2- to 3.5-fold gain in luciferase expression observed in the examined cells in comparison to the one-step system. Interestingly, the conventional TSTA system suppressed the gene expression in comparison to the one-step system. A significant enhancement of expression by the advanced TSTA was indicated by the 4- to 15 -fold gain of luciferase expression in comparison to the conventional system (Fig. 2C). Therefore, the advanced TSTA system significantly improved hTERT-dependent gene expression in all of the cancer cell lines examined in comparison to both of the previous systems. These results demonstrate that there is a strong transcriptional amplification exerted by the GAL4-VP16 fusion protein with the polyglutamines and the rat glucocorticoid receptor in the hTERT-dependent cancer-specific gene expression.

The fold gain achieved by the advanced TSTA system was calculated by comparing the luciferase expression between the one-step and advanced TSTA system. Interestingly, the regression analysis showed a significant correlation between the telomerase activity of the cells and the fold gain of luciferase expression (Fig. 2B). Therefore, the advanced TSTA system enabled a higher fold gain of gene expression as telomerase activity increases in the cancer cell lines. These results indicate that the advanced TSTA system further improves the one-step system in cancer cells with higher telomerase activity.

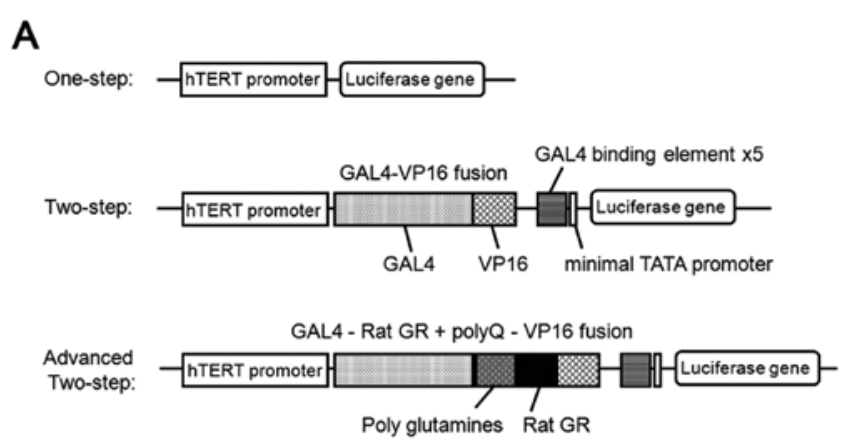

B

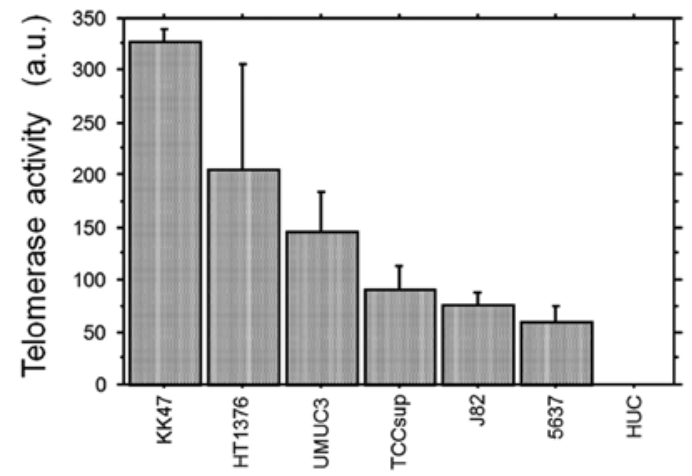

C

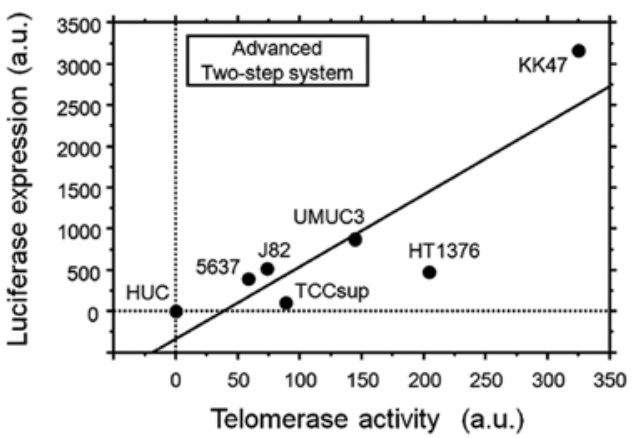

Figure 1. (A) A schematic diagram of the advanced TSTA (two-step transcriptional amplification) system is shown in the bottom panel. An hTERT promoter is used for the cancer-specific gene transcription. The first step of the conventional TSTA system (middle panel) includes the sequence of the GAL4-VP16 fusion protein. In the second step, the expressed GAL4-VP16 protein drives the target gene expression under the control of GAL4 response elements (GAL4 binding sites) upstream of the minimal promoter. In the advanced TSTA system (bottom panel), the polyglutamines and rat glucocorticoid receptor (GR) sequences are inserted between the GAL4 and VP16 sequences to enhance luciferase gene expression. (B) Telomerase activity was determined in a variety of human bladder cancer cell lines and normal human bladder urothelial cells (HUC). The data are shown as arbitrary unit (a.u.) and from triplicate measurements. (C) The relationship between the telomerase activity (a.u.) and luciferase expression (a.u.) in the advanced TSTA system was examined by a regression analysis. The level of luciferase expression was determined by the luminescence of luciferin, a substrate for luciferase. The analysis showed a significant association between the parameters $(\mathrm{p}<0.05)$.

Detection of human bladder cancer cells with the advanced TSTA mediated cancer-specific gene expression. A green fluorescent protein (GFP) gene encoding plasmid vector was constructed with the hTERT promoter-driving advanced TSTA to utilize the system in imaging and detection of viable bladder 
A
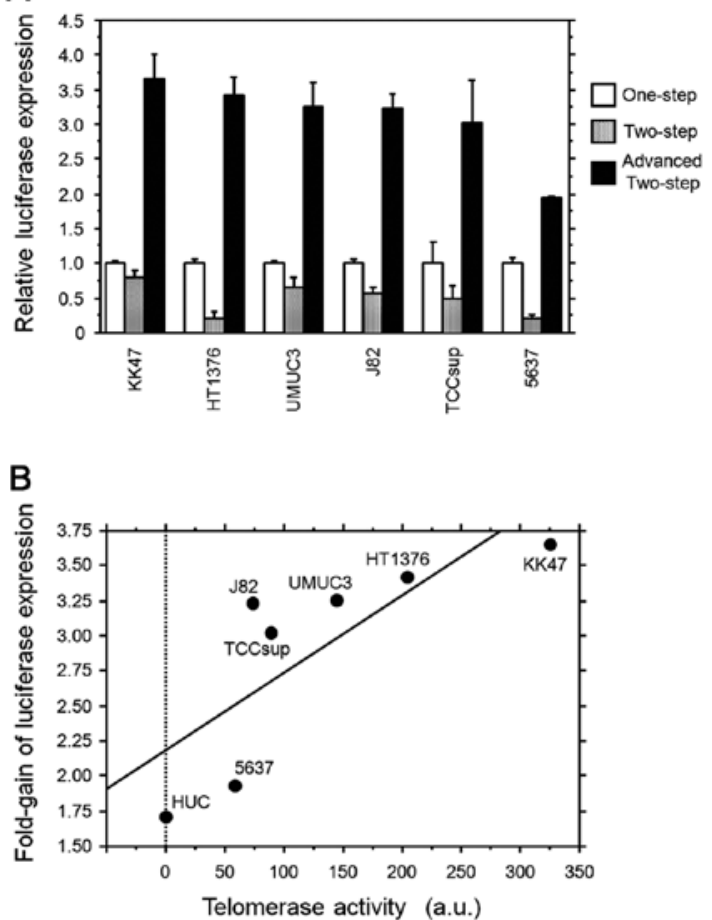

C

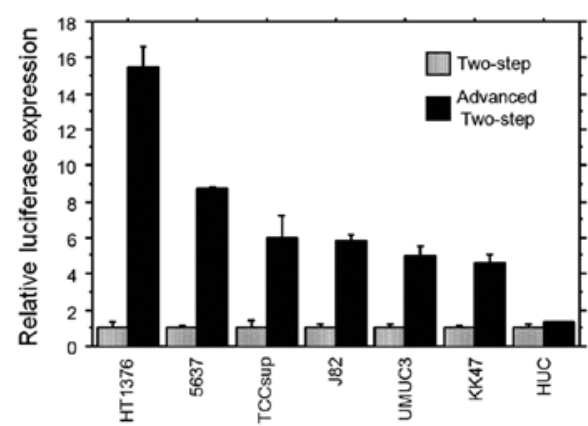

Figure 2.(A) The in vitro gene expression was compared between the one-step, conventional TSTA and advanced TSTA systems. The luciferase expression of the one-step system was defined as 1.0, and the relative luciferase expression ratios of the conventional and advanced TSTA systems are presented for each cell line in comparison to the one-step system. The data are from triplicate measurements. (B) The relationship between the telomerase activity (a.u.) and fold gain of luciferase expression in the advanced TSTA was examined by a regression analysis. The $y$-axis value of (A) for the advanced TSTA in the cell lines is used as the fold gain of the luciferase expression here. The analysis showed a significant association between the parameters $(\mathrm{p}<0.05)$. (C) The in vitro gene expression was compared between the conventional TSTA and advanced TSTA systems. The luciferase expression of the conventional TSTA system was defined as 1.0, and the relative luciferase expression ratios of the advanced TSTA system are presented for each cell line in comparison to the conventional TSTA system. The data are from triplicate measurements.

cancer cells. The advanced TSTA-hTERT-GFP plasmid successfully induced cancer-specific gene expression, showing robust GFP expression in human bladder cancer cell lines, but no visible GFP expression in normal bladder urothelial cells (Fig. 3A and B). The control GFP plasmid with CMV promoter induced GFP expression in both normal bladder cells (Fig. 3A) and cancer cells (data not shown).

An in vitro experiment using human bladder cancer, KK47 cells was done to further test the efficacy of the plasmid system
A

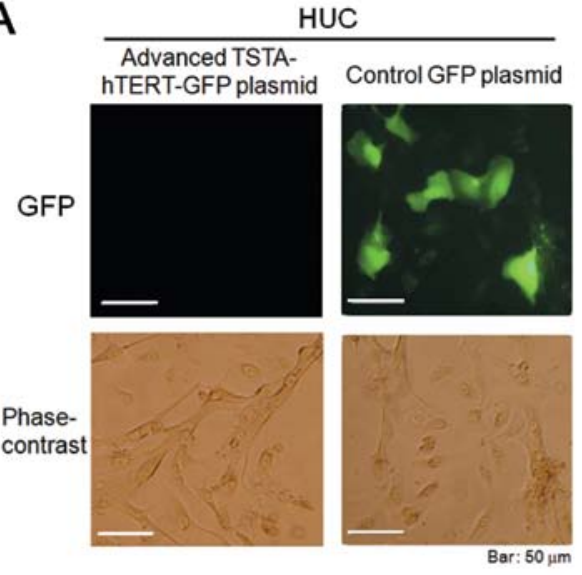

B

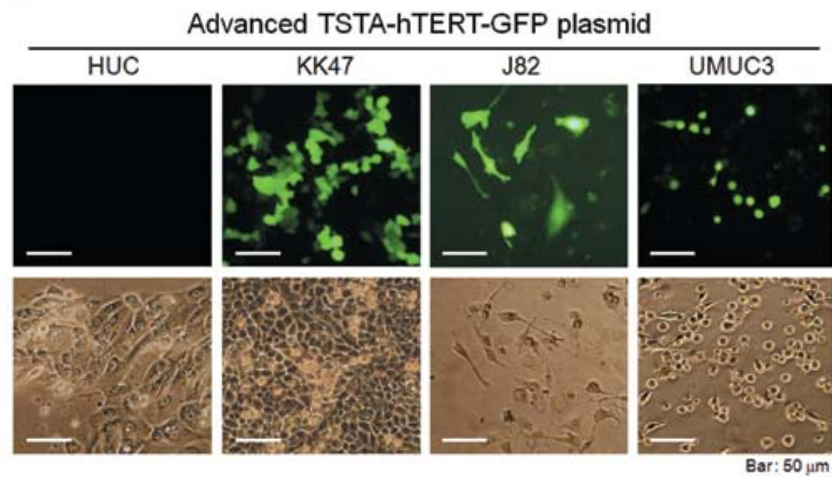

Figure 3. (A) The advanced TSTA-hTERT-GFP plasmid is constructed for the cancer cell-imaging by green fluorescent protein (GFP) expression, in which hTERT dependent cancer-specific gene expression is enhanced by the advanced TSTA system. The advanced TSTA-hTERT-GFP plasmid does not induce any visible GFP expression in normal human bladder urothelial cells (HUC). The GFP expression was observed in the normal bladder cells transfected with the control GFP plasmid with the CMV promoter. (B) The advanced TSTA-hTERT-GFP plasmid induces cancer-specific GFP expression. Robust GFP expression is observed in human bladder cancer cell lines (KK47, J82, UMUC3), but not visible in the normal human bladder urothelial cells.

for bladder cancer cell detection (Fig. 4A). KK47 cancer cells were mixed with normal human bladder urothelial cells (HUC) and these cells were transfected with the advanced TSTA-hTERT-GFP plasmid. GFP expression of these cells was examined after $24 \mathrm{~h}$ under fluorescent microscopy. The KK47 cancer cells were pretreated with the Cell Tracker Orange as a tracer to ensure that the cells expressing the GFP signals were indeed the target KK47 cancer cells. Dual fluorescent microscopy confirmed that most of GFP-positive cells were KK47 bladder cancer cells labeled with Cell Tracker Orange in the cultured cells with different target frequency ratios (1:10 and 1:100) (Fig. 4B). The cancer cells could not be distinguished from the other normal bladder cells morphologically in the bright fields.

\section{Discussion}

Imaging of cancer-specific gene expression depends on robust levels of the products at both the mRNA and protein levels. Although the hTERT promoter is used as a broad ranged cancer-specific promoter $(3,4,7-9)$, the gene expression driven 
A
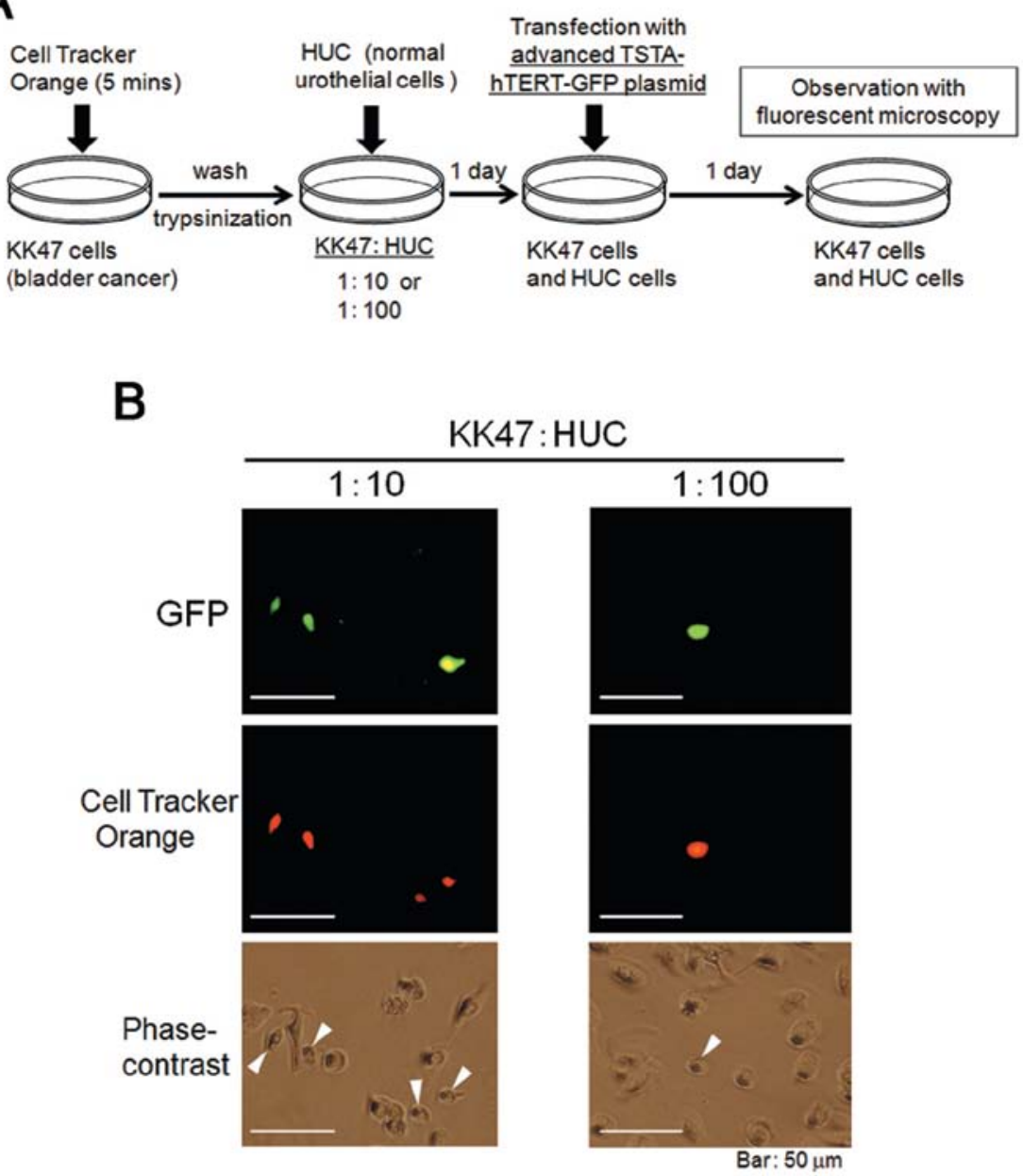

Figure 4. (A) Selective visualization of human bladder cancer cells by using the advanced TSTA-hTERT-GFP plasmid. A schematic illustration of the experimental procedure is shown. The KK47 human bladder cancer cells were pretreated with the Cell Tracker Orange as a tracer to ensure that the cells expressing GFP signals are indeed the target KK47 cancer cells. The target KK47 cancer cells were mixed with normal human bladder urothelial cells (HUC) and the cells were incubated for $24 \mathrm{~h}$. The mixed bladder cells were then transfected with the advanced TSTA-hTERT-GFP plasmid. GFP expression and Cell Tracker Orange tracer in the cancer cells was directly examined under fluorescent microscopy. (B) Representative images of the co-cultured cells are shown at the indicated frequency ratio of target KK47 bladder cancer cells (1:10 and 1:100) for GFP expression, Cell Tracker Orange under fluorescent microscopy. Most of GFP-positive cells were confirmed to be KK47 bladder cancer cells labeled with Cell Tracker Orange and viable KK47 cancer cells were easily visualized among the normal human bladder urothelial cells. In the bright fields, the cancer cells are indicated by an arrowhead.

by the hTERT promoter is often weak due to its poor transcriptional activity (9-11). This study described a novel strategy to enhance the cancer-specific gene expression and the imaging driven by hTERT promoter and overcome the weak points of the promoter. The advanced two-step transcriptional amplification (TSTA) system was developed by putting the polyglutamines and rat GR sequences within the GAL4-VP16 fusion sequence of the conventional TSTA system. The advanced system strongly activated the hTERT promoter-driven reporter gene expression in the expected cancer-specific manner based on the telomerase activity.

The advanced TSTA system-mediated amplification of luciferase expression was $\sim 3.5$-fold higher than the one-step system in the bladder cancer cell lines. Furthermore, significant enhancement was also obtained $(\sim 15$-fold) in a comparison between the advanced TSTA system and the conventional TSTA system. Interestingly, the hTERT-driven expression was moderately suppressed by the conventional TSTA system in comparison to the one-step system in the bladder cancer cell lines. Therefore, the hTERT-driven advanced TSTA enabled the enhancement of cancer-specific gene expression in the examined bladder cancer cell lines in comparison to both the one-step and conventional TSTA systems.

The current study clearly indicates that the advanced system with the polyglutamines and rat GR insert functions under the hTERT promoter. In addition, a greater fold gain in luciferase gene expression was observed in the advanced TSTA system as telomerase activity was elevated in bladder cancer cells. The findings indicate that the advanced TSTA system improves the one-step system in the bladder cancer cells with higher hTERT activity. The mechanism underlying this effect is that the polyglutamines and rat GR region significantly increase the levels of the GAL4-VP16 fusion protein, thereby increasing the ability to bind to GAL4 binding sites upstream of the second minimal promoter, thus resulting in higher levels of mRNA transcription and subsequent protein expression. 
The hTERT promoter-driving advanced TSTA system was developed as a tool for the diagnosis of bladder cancer, since there was a significant up-regulation of the luciferase expression using the advanced TSTA expression system. The advanced TSTA-hTERT-GFP plasmid was constructed and the in vitro utility of the plasmid system for the detection of bladder cancer cells was evaluated. The advanced TSTA-hTERT-GFP plasmid successfully induced cancer-specific gene expression, showing robust GFP expression in bladder cancer cells, but no visible GFP expression in normal urothelial cells. There was selective visualization of viable human bladder cancer cells in the cultured cells containing a mixture of 10- and 100-fold more normal bladder cells. These results demonstrate that the advanced TSTA-hTERT-GFP plasmid could be a useful imaging tool in in vitro where the detection and/or selection of viable cancer cells are required. In the absence of the advanced TSTA system, GFP expression was close to background in the bladder derived normal and cancer cells, and therefore, the cancer cells could not be selectively imaged under fluorescent microscopy.

This study validated a novel TSTA system that robustly enhances cancer-specific gene expression in human bladder cancer cells. The advanced TSTA-hTERT expressional system should be a valuable tool for detecting viable bladder cancer cells in urine and could be applied for in vitro detection of various types of cancer cells disseminated in vivo. It would therefore be of particular interest to extend this strategy to clinical practice and to confirm the efficacy of the system as a non-invasive method for the diagnosis and evaluation of bladder cancer.

\section{Acknowledgements}

This study was supported by a grant from the Ministry of Education, Culture, Sports, Science and Technology's FY2006 'Creation of Innovation Centers for Advanced Interdisciplinary Research Areas' Scheme in Japan.

\section{References}

1. Kirkali Z, Chan T, Manoharan M, Algaba F, Busch C, Cheng L, Kiemeney L, Kriegmair M, Montironi R, Murphy WM, Sesterhenn IA, Tachibana M and Weider J: Bladder cancer: epidemiology, staging and grading, and diagnosis. Urology 66: 4-34, 2005.
2. Takakura M, Kyo S, Kanaya T, Hirano H, Takeda J, Yutsudo M and Inoue M: Cloning of human telomerase catalytic subunit (hTERT) gene promoter and identification of proximal core promoter sequences essential for transcriptional activation in immortalized and cancer cells. Cancer Res 59: 551-557, 1999.

3. Kishimoto H, Kojima T, Watanabe Y, Kagawa S, Fujiwara T, Uno F, Teraishi F, Kyo S, Mizuguchi H, Hashimoto Y, Urata Y, Tanaka $\mathrm{N}$ and Fujiwara T: In vivo imaging of lymph node metastasis with telomerase-specific replication-selective adenovirus. Nat Med 12: 1213-1219, 2006

4. Maida Y, Kyo S, Sakaguchi J, Mizumoto Y, Hashimoto M, Mori N, Ikoma T, Nakamura M, Takakura M, Urata Y, Fujiwara T and Inoue M: Diagnostic potential and limitation of imaging cancer cells in cytological samples using telomerase-specific replicative adenovirus. Int J Oncol 34: 1549-1556, 2009.

5. Lin Y, Miyamoto H, Fujinami K, Uemura H, Hosaka M, Iwasaki Y and Kubota Y: Telomerase activity in human bladder cancer. Clin Cancer Res 2: 929-932, 1996.

6. Xie XY, Yang X, Zhang JH and Liu ZJ: Analysis of hTERT expression in exfoliated cells from patients with bladder transitional cell carcinomas using SYBR green real-time fluorescence quantitative PCR. Ann Clin Biochem 44: 523-528, 2007.

7. Huang P, Watanabe M, Kaku H, Kashiwakura Y, Chen J, Saika T, Nasu Y, Fujiwara T, Urata Y and Kumon H: Direct and distant antitumor effects of a telomerase-selective oncolytic adenoviral agent, OBP-301, in a mouse prostate cancer model. Cancer Gene Ther 15: 315-322, 2008.

8. Huang P, Kaku H, Chen J, Kashiwakura Y, Saika T, Nasu Y, Urata Y, Fujiwara T, Watanabe $M$ and Kumon H: Potent antitumor effects of combined therapy with a telomerasespecific, replication-competent adenovirus (OBP-301) and IL-2 in a mouse model of renal cell carcinoma. Cancer Gene Ther 17: 484-491, 2010

9. Watanabe M, Ueki H, Ochiai K, Huang P, Kobayashi Y, Nasu Y, Sasaki K, Kaku H, Kashiwakura Y and Kumon H: Advanced two-step transcriptional amplification as a novel method for cancer-specific gene expression and imaging. Oncol Rep 26: 769-775, 2011.

10. Sadowski I, Ma J, Triezenberg S and Ptashne M: GAL4-VP16 is an unusually potent transcriptional activator. Nature 335 : 563-564, 1988.

11. Iyer M, Wu L, Carey M, Wang Y, Smallwood A and Gambhir SS: Two-step transcriptional amplification as a method for imaging reporter gene expression using weak promoters. Proc Natl Acad Sci USA 98: 14595-14600, 2001.

12. Gerber HP, Seipel K, Georgiev O, Höfferer M, Hug M, Rusconi S and Schaffner W: Transcriptional activation modulated by homopolymeric glutamine and proline stretches. Science 263: 808-811, 1994.

13. Zhang K, Watanabe M, Kashiwakura Y, Li SA, Edamura K, Huang P, Yamaguchi K, Nasu Y, Kobayashi Y, Sakaguchi M, Ochiai K, Yamada H, Takei K, Ueki H, Huh NH, Li M, Kaku H, $\mathrm{Na} \mathrm{Y}$ and Kumon H: Expression pattern of REIC/Dkk-3 in various cell types and the implications of the soluble form in prostatic acinar development. Int J Oncol 37: 1495-1501, 2010.

14. Emami KH and Carey M: A synergistic increase in potency of a multimerized VP16 transcriptional activation domain. EMBO J 11: 5005-5012, 1992. 